\title{
ENGAJAMENTO CORPORATIVO COM STARTUPS: SÍNTESE E ANÁLISE CRÍTICA DA LITERATURA
}

Raoni Barros Bagno (ㅁbagno@ dep.ufmg.br) - Universidade Federal de Minas Gerais (UFMG)

Mario Sergio Salerno (msalerno@usp.br) - Universidade de São Paulo (USP)

Wesley Canedo de Souza Junior (wesleycanedo@gmail.com) - Universidade Federal de Minas Gerais (UFMG)

\section{RESUMO}

Engajamento Corporativo com Startups (ECcS) se trata de uma iniciativa de inovação aberta em que grandes empresas interagem com startups visando melhoria de desempenho em inovação. Complementaridades entre empresas de grande porte (e.g. disponibilidade de recursos, inércia para inovar...) e startups (escassez de recursos, flexibilidade, tecnologias de ponta...) estão na base destes engajamentos. Contudo, grandes empresas e startups possuem diferenças marcantes, de forma que o trabalho conjunto entre elas traz muitos desafios e, especialmente para a grande empresa, muitas questões internas à organização emergem ao se buscar interagir com um negócio nascente (e.g. processos, aspectos culturais, compartilhamento de recursos e informação protegida, etc.). O debate corrente na temática de ECcS negligencia, em grande extensão, a complexidade subjacente aos elementos internos à grande organização para propiciar engajamentos efetivos com startups. Estudos que tangenciam a questão se limitam frequentemente a mencionar um Champion que atue expandindo limites organizacionais, dando suporte ao trabalho com startups. Dessa forma, o presente estudo sintetiza as abordagens da atual literatura sobre ECcS, identificando seus antecedentes e vertentes principais. Na sequência, o texto aborda a lacuna entre o debate corrente e as questões organizacionais no campo da gestão da inovação, levantando implicações e direções para estudos em ECcS.

Palavras-chave: Engajamento Corporativo com Startups; Inovação Aberta; Gestão da Inovação; Inovação sistemática

\section{INTRODUÇÃO}


A inovação tem sido largamente aceita como um caminho mandatório para o alcance e manutenção da competitividade de empresas (TIDD; BESSANT; PAVITT, 2001; AdAMS; BESSANT; PHELPS, 2006; O'CONNOR et al., 2008). Especialmente para empresas estabelecidas de grande porte, esforços para se alcançar desempenho em inovação estão em constante conflito com as demandas por excelência operacional e melhorias de curto prazo (DUNCAN, 1976; TIDD; Bessant; PAvitt, 2001; O'Connor et al., 2008; O'Reilly III; Tushman, 2013). Ao longo das últimas décadas este tem sido provavelmente o desafio central no campo da gestão da inovação em grandes empresas.

O conceito de Inovação Aberta, cunhado por Chesbrough (2003), se tornou muito influente neste campo. Neste, uma empresa experimenta fluxos de conhecimento de dentro para fora (outbound) ou de fora para dentro (inbound) em seu processo de inovação, permitindo-lhe reduzir riscos e incertezas associados à inovação (CHESBROUGH, 2003; HUIZINGH, 2011). Não obstante, mesmo que a inovação aberta permita inovar a partir de ideias, recursos e expertise externos, a construção de uma capacidade de inovar abertamente resulta de um longo processo de mudança em uma organização (e.g. ChIARONI; ChIESA; FratTINI, 2011) e há muitos alertas provindos da literatura em relação aos riscos de se terceirizar capacidades de inovação da empresa (c.f. HUIZINGH, 2011). Portanto, ao invés de evitar transformações organizacionais, a prática da inovação aberta envolveria também mudanças organizacionais significativas.

Os Engajamentos Corporativos com Startups (ECcS), em grande parte motivados por um recente avanço e difusão das tecnologias digitais, têm emergido como uma forte e promissora modalidade de inovação aberta. Muitos estudos reportam crescentes números de empresas que tem buscado efetivar algum tipo de interação com startups (WEIBLEN; CHESBROUGH, 2015; Kohler, 2016; Livieratos; LePENIOTIS, 2017; Prashantham; Kumar, 2019). ECcS podem assumir uma ampla gama de formatos, finalidades e níveis de interação. De forma geral, para empresas estabelecidas, ECcS surgem como uma forma de se mitigar incertezas e inovar mais radicalmente; para as startups, possibilitam acesso a recursos e impulso extra para o crescimento de seus negócios.

Literatura específica no campo de ECcS é ainda inicial e bastante dedicada ao entendimento das estratégias dos programas corporativos, do processo de engajamento e de recomendações sobre como selecionar parceiros (HoGENHUIS; VAN DEN Hende; HUlTinK, 2016; SPENDER et al., 2017; Selig; GASSER; BALtes, 2018). Como uma grande empresa se organiza para engajamentos sistemáticos com startups é algo fracamente abordado, mesmo em face dos 
numerosos desafios organizacionais subjacentes ao desenho e gestão de parcerias entre grandes empresas e startups - fortemente assimétricas dadas as inúmeras diferenças entre tais parceiros. O presente estudo objetiva sintetizar as abordagens da atual literatura sobre ECcS, identificando seus antecedentes e vertentes principais. Na sequência, o texto aborda a lacuna entre o debate corrente e as questões organizacionais, levantando implicações e direções para estudos em ECcS da perspectiva da empresa estabelecida.

\section{MÉTODO}

O levantamento bibliográfico foi realizado inicialmente nas plataformas Sciencedirect e Ebsco, Business Source Complete em abril de 2019. Após lapidação inicial, foi aplicada a seguinte expressão booleana: (startup or start-up or start up or startups or start-ups) AND (engagement or partnership or agreement or acceleration or incubation). Complementarmente, a primeira parte da expressão foi combinada na função AND com: (company or companies or mnc or firm or corporate). Após um primeiro filtro de títulos e de resumos, foram obtidos 19 artigos. Expressões similares foram testadas no Power Search (plataforma do Babson College que combina diversas bases de dados) e também no WebofKnowledge sem, no entanto, agregar novos textos acadêmicos em relação à busca inicial, porém contribuindo com relatórios e relatos gerenciais que expressam o pensamento sobre o tema no campo das práticas.

Tendo identificado os trabalhos de Weiblen e Chesbrough (2015) e de Kohler (2016) como centrais para o debate corrente em ECcS, retornamos às bases buscando textos recentes que citam algum destes estudos, observadas as afinidades de título/resumo. Somado à identificação de referências relevantes nos estudos previamente selecionados ("bola de neve"), obteve-se a lista final de 48 textos que compuseram as referências deste levantamento nas temáticas de ECcS e adjacências (por demanda de síntese, nem todos citados no presente artigo).

Subsequentemente, os textos foram agrupados por afinidades e vertentes, inspirando os tópicos da próxima seção. Ratificada a lacuna de estudos com foco nas implicações organizacionais de $\mathrm{ECcS}$, o debate da seção 4 foi proposto bom base em investigações anteriores (e.g. BAGNO; SAlERNO; Dias, 2017; BAGNO; SAlerno; Silva, 2017; Melo; Bagno, 2017).

\section{ANTECEDENTES DOS ECCS}




\subsection{Iniciativas de Corporate Venturing e sua integração com a Inovação Aberta}

Iniciativas de Corporate Venturing (CV), ou mais especificamente programas de Corporate Venture Capital (CVC) conduzidos por empresas de grande porte, constituem provavelmente os antecedentes mais influentes do debate atual em ECcS. Em seu período inicial, entretanto, investimentos de $\mathrm{VC}$ poderiam ser mais bem associados com aquisição direta de tecnologia (i.e. realizadas fora do ambiente interno da empresa e com pouca ou nenhuma interação com este) ou até mesmo de negócios inteiros. Ainda assim, iniciativas de CVC são consideradas uma fonte de renovação da estratégia organizacional e de exploração de novas oportunidades de negócio (e.g. BASU; PHELPS; KOTHA, 2011). Weiblen e Chesbrough (2015) consideram que o capital de risco corporativo, juntamente com as incubadoras corporativas, sejam os modelos mais bem estabilizados de engajamento de grandes empresas com startups devido à sua difusão e ao tempo de maturação destas abordagens no mercado.

Livieratos e Lepeniotis (2017) argumentam por uma quarta onda de CVC, na qual os programas estão cada vez mais integrados com a abordagem de inovação aberta das empresas e, dessa forma, passam a ser complementados por inúmeras outras iniciativas provindas desta vertente.

\subsection{Empreendedorismo Corporativo e Ambidestria Organizacional}

O Empreendedorismo Corporativo, conceito cunhado no campo dos estudos de estratégia, habilitaria as empresas a extrair valor incremental de seus negócios correntes e, simultaneamente, lançarem-se em oportunidades de inovação promissoras (SELIG; GASSER; BALTES, 2018; SHANKAR; SHEPHERD, 2018). Essa ideia central é similar àquela presente nos estudos de ambidestria organizacional, que tem suas raízes em estudos organizacionais e postula que uma empresa precisa "explotar" seu negócio corrente (i.e. aumentar a eficiência, inovar incrementalmente dentro de seus domínios) e, ao mesmo tempo, "explorar" oportunidades de ruptura (inovação radical, fora de seus domínios) (O'REILly; TUSHMAN, 2004; RAISCH; BIRKINSHAW, 2008).

Dada a vocação das estruturas estabelecidas para realizar o eploitation, Alänge e Steiber (2018) afirmam que a realização de parcerias com startups seria uma forma de resolver o desafio da ambidestria em grandes empresas ao trazer o "exploration" para a rotina interna da organização. Estes autores oferecem um framework de modos operacionais para ambidestria baseados em experiências de sucesso de grandes empresas de manufatura em sua interação com empresas nascentes de base tecnológica com propósitos de inovação. 


\subsection{Parcerias Assimétricas}

Parcerias assimétricas são consideradas aquelas em que os parceiros diferem entre si de maneira significativa, seja em termos de porte, recursos ou experiência comercial (MinshALL et al., 2010). Este é exatamente o caso quando empresas estabelecidas e startups trabalham juntas.

A expropriação de recursos do lado fraco de uma parceria é um problema central discutido na linha de estudos de parcerias assimétricas. Freytag (2019) assume que startups e empresas estabelecidas somente podem ser bem-sucedidas em parcerias de longo prazo se os interesses de ambas as partes forem adequadamente considerados - o que não seria necessariamente óbvio no contexto de empresas estabelecidas em cadeias de valor também consolidadas. Longas trajetórias em relações interinstitucionais verticalizadas marcadas por governança centralizada da rede, uso constante de poder de barganha e de contratos formais estritamente detalhados são fatores marcantes no contexto de relações interinstitucionais de muitas empresas de grande porte (HumPhreY; SCHMitZ, 2000; BAGNO et al., 2012). Apesar das considerações e advertências provenientes dessa linha, Minshall et al. (2010) reforçam que parcerias assimétricas entre startups e empresas de grande porte podem prover vantagens econômicas relevantes para ambos.

\section{ENGAJAMENTOS CORPORATIVOS COM STARTUPS}

Weiblen e Chesbrough (2015) e Kohler (2016) podem ser considerados como estudos pioneiros das formas modernas de ECcS. De acordo com Kohler (2016), empresas se engajam com startups para desenvolver novos produtos e serviços juntas, explorar novas oportunidades de mercado ou para compartilhar tecnologia e talentos para resolver desafios de negócios. Para uma startup, a grande empresa pode ser um parceiro importante para testar sua adequação produto-mercado, escalonar operações, oferecer suporte à distribuição, levantar fundos ou mesmo atrair maior credibilidade ao negócio nascente por apoiar-se em uma marca bem estabelecida (KoHLER, 2016). Adicionalmente, Lawton (2019) destaca que o engajamento com startups pode ajudar empresas estabelecidas a prospectar o futuro de sua indústria por meio da lente dos empreendedores, mudar a cultura de sua organização em favor de um comportamento ágil e reduzir ineficiências e custos gerais em processos. Kaplan (2019) conclui que, apesar de grandes empresas e startups poderem ser consideradas como mundos totalmente diferentes, seu casamento pode ser o caminho para a inovação sustentável. 
As formas pelas quais uma empresa estabelecida pode interagir com startups são numerosas. Spender et al. (2017) citam diferentes mecanismos adotados como CVC, incubadoras internas, alianças estratégicas e joint ventures. Lawton (2019), além dos programas corporativos de aceleração, cita os bootcamps, patrocínio de empreendedores e engajamentos diretos com startups de destaque previamente identificadas em uma indústria específica. Por sua vez, Minsky (2019) faz referência a aceleradoras corporativas, cooperação direta com startups e investimentos e/ou aquisição de startups. Já Kohler (2016) classifica os engajamentos em hackatons corporativos, incubadoras de negócios, incubadoras corporativas, corporate venturing e fusões e aquisições. Weiblen e Chesbrough (2015) identificam quatro tipos básicos de engajamentos entre empresas de grande porte e startups, resultantes do cruzamento entre as variáveis de fluxo de inovação e envolvimento em equity (Figura 1): Corporate Venturing, Incubadora Corporativa, programas Ouside-In e Plataforma (programas inside-out).

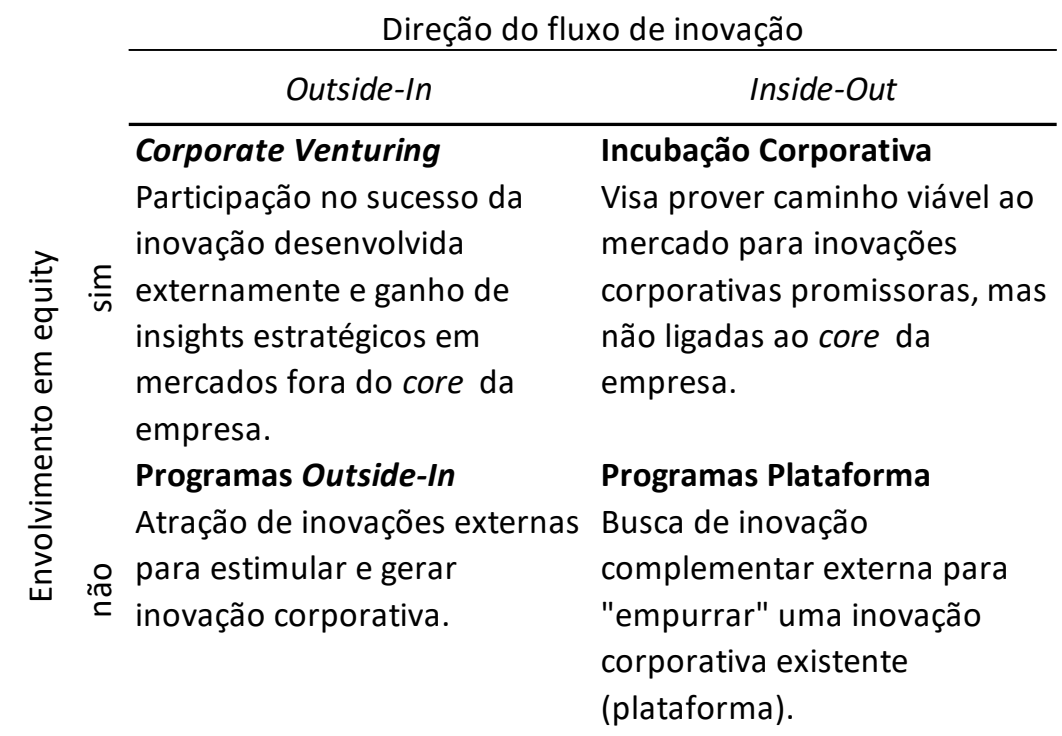

Figura 1 - Tipos de ECcS. Adaptado de Weiblen e Chesbrough (2015)

Ainda que o número crescente de iniciativas e estudos em ECcS possam abrir várias possibilidades no campo da inovação e do empreendedorismo, o entusiasmo com essa abordagem é ponderado também por casos de insucesso. Engajar grandes empresas com startups guarda desafios complexos subjacentes ao discurso dominante de seus benefícios e pesquisas recentes relevam que muitos dos esforços realizados não alcançam as expectativas e são abandonados (WeIBlen; ChESBrough, 2015; PrashanthaM; YiP, 2016). Minshall et al. (2010) alertam sobre os inúmeros desafios gerenciais para a correta implantação e gestão de parcerias entre grandes empresas e startups. Esforços especiais são necessários para forjar estes 
relacionamentos e fazê-los funcionar de fato. Assim, como construir uma capacitação organizacional para realizar sistematicamente parcerias com startups torna-se um desafio relevante (INNOVATION LEADER, 2019; PRASHANTHAM; KUMAR, 2019).

A Figura 2 oferece um mapa conceitual do debate até então delineado em ECcS e localiza a lacuna de interlocução com o campo da gestão sistemática de inovação - campo pouco tocado entre os antecedentes identificados desta discussão e que oferece base conceitual para de se avançar no "como" organizar grandes empresas para ECcS.

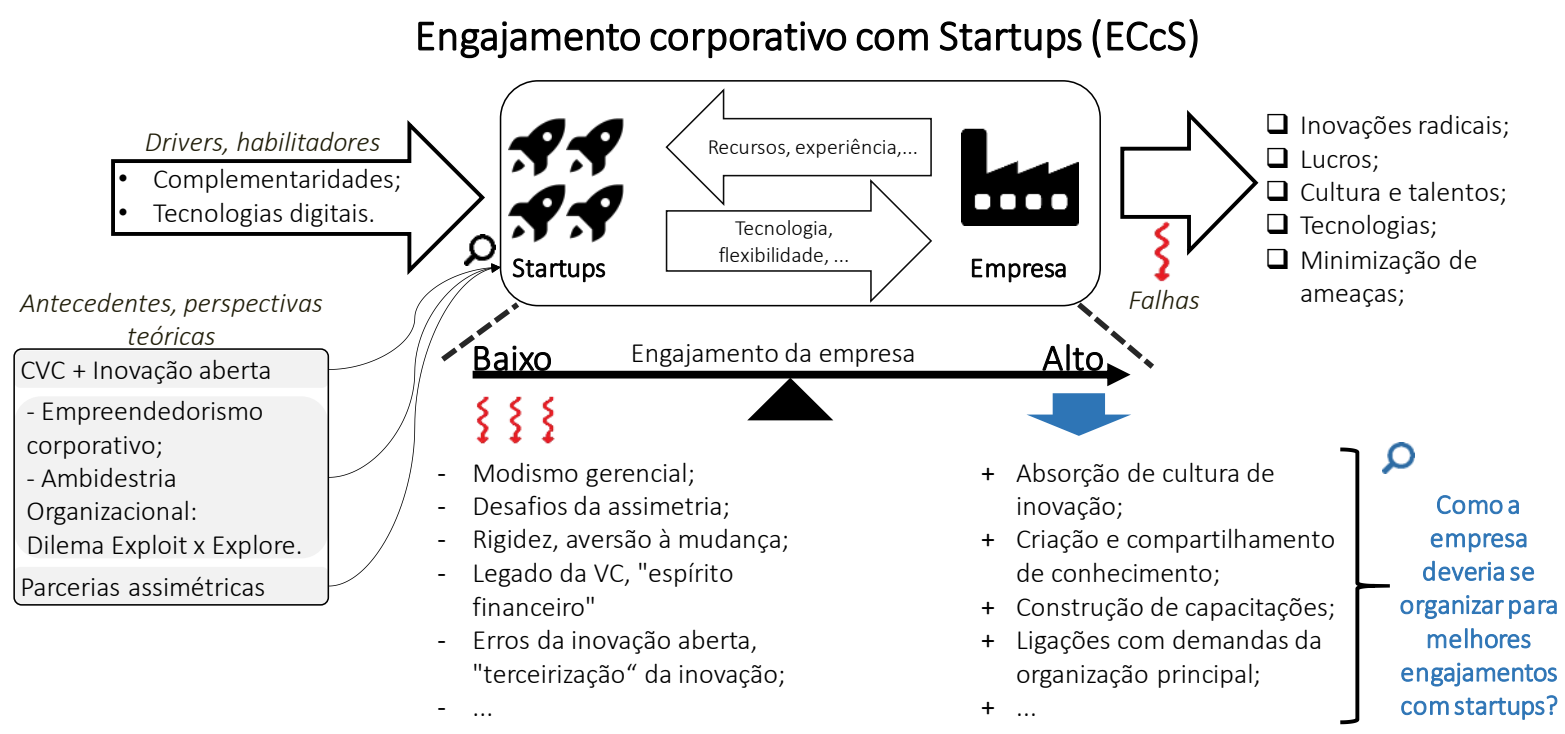

Figura 2 - Mapa conceitual do debate em ECcS e gap de interlocução com estudos no campo da Gestão sistemática de inovação tecnológica. Fonte: dos autores.

\section{ANÁLISE CRÍTICA E IMPLICAÇÕES}

\subsection{Vieses dos estudos (e das práticas?) em ECcS: tópicos de gestão e organização da grande empresa negligenciados}

Selig, Gasser e Baltes (2018) notam que as implicações externas de uma abertura do processo empresarial de inovação ganharam muita atenção nos últimos anos, ao passo que os aspectos internos de tal abertura ainda carecem de maiores investigações. Em geral, a literatura em ECcS não foca em aspectos organizacionais consequentes destes engajamentos e avancem além das questões imediatas associadas ao design e operação dos programas de startups. Exemplos disso podem ser observados nas declarações de objetivos de estudos como os de Kohler (2016), 
Richter, Jackson e Schildhauer (2018), Shankar e Shepherd (2018) ou Kanbach e Stubner (2016).

Os problemas em se considerar insuficientemente os aspectos organizacionais de ECcS ecoam os argumentos de Greenwood e Miller (2010) ao colocarem que o design organizacional tem sido fortemente negligenciado nos últimos anos a despeito de sua importância crítica para o desempenho das organizações. Especialmente no campo da gestão da inovação, uma ênfase desproporcional nos processos gerenciais teria encoberto as complexidades de se construir sistemas de gestão e estruturas organizacionais que catalisassem a ocorrência de inovação sistemática em grandes empresas (O'CONNOR, 2012).

De acordo com Mahmoud-Jouini, Duvert e Esquirol (2018), um baixo nível de engajamento e atração de recursos do ambiente interno da própria organização impediria que boas startups fossem também atraídas. Portanto, ser um parceiro seguro e atrativo seria fundamental não apenas para o sucesso de ECcS em si, mas também para a conexão destas iniciativas com outros processos e ações na empresa. Quando recursos são imóveis e os incentivos desalinhados, o processo de inovação desacelera (FREEMAN; ENGEL, 2007).

\subsection{Muita coisa para um champion... a orquestração dos ECcS e estruturas organizacionais subjacentes}

Alguns estudos dão certa ênfase ao papel gerencial de condução da interface entre a empresa e as startups ao identificarem um "champion influente" para liderar a iniciativa (INNOVATION LEADER, 2019) ou ao considerarem o suporte dado por "desenvolvedores de negócios dedicados" (c.f. MAHMOUD-JouINI; DUVERT; EsQUIROL, 2018) que atuariam como hubs ou agentes de abertura das portas da organização em prol dos relacionamentos com startups. Kohler (2016) coloca os gestores de aceleradoras corporativas como os elementos de ligação entre startups e corporações ao proverem às startups acesso às pessoas certas dentro da organização e assegurar que as inovações desenvolvidas externamente sejam usadas internamente. Para isso, tais pessoas deveriam ter uma capacidade de trabalhar com startups e simultaneamente navegar bem nas estruturas organizacionais. Nossa análise é que as expectativas são bastante altas em torno deste gestor.

Como observado por Freeman e Engel (2007), formas orgânicas de inovação em grandes organizações são difíceis de implementar e normalmente contam com esforços heroicos de 
poucos indivíduos que lutam constantemente contra muitas barreiras internas (e.g. culturais, políticas, disputa por recursos), hierarquias e rigidez excessivas em processos e normas (Kelley, 2009; Arena et al., 2017; O'Connor; Corbett; Peters, 2018). Minsky (2019) chega a argumentar que as empresas fazem um "teatro da inovação" ao dizerem que estão atentas ao cenário de startups, mas não terem praticamente nenhuma ação concreta em prática. Em relação à estrutura de trabalho, Lawton (2019) clama pela necessidade de times dedicados na empresa para trabalharem com os novos parceiros e reforça que educar os stakeholders internos nessa direção demanda imenso esforço. Em última análise, o autor afirma que o tempo e atenção que os executivos de empresas estabelecidas realmente direcionam ao engajamento com startups ditam o valor de toda a iniciativa.

\subsection{Nível de engajamento em ECcS e a tensão entre separação e integração}

A maior parte das iniciativas reportadas nos estudos selecionados descreveram programas relacionados a ECcS que rodaram fisicamente separados da organização principal (e.g. Livieratos; LePeniotis, 2017; Selig; Gasser; Baltes, 2018; Prashantham; Kumar, 2019). De fato, estruturas separadas permitiriam maior autonomia aos programas de startups e evitariam conflitos com as estruturas organizacionais complexas das grandes organizações (KOHLER, 2016). Tal cenário, contudo, contrasta com determinadas expectativas normalmente associadas aos ECcS como a de oferecer transbordamentos culturais e comportamentais à empresa, influenciar seus processos internos, integrar o talento das pessoas de startups, alimentar a estratégia da empresa ou ainda estabelecer conexões regulares entre as startups e diferentes funções internas à organização.

Em geral, formas programáticas tenderiam a rodar o processo de inovação em separado das redes informais internas necessárias para a adaptação e suporte à inovação - um conflito bem estabelecido na literatura de ambidestria organizacional e com raras interações com as questões típicas dos ECcS. Dessa forma, as inovações vem a ser desenvolvidas fora do ecossistema social da organização e podem ser debilitadas ao se defrontarem com os desafios de integração (ARENA et al., 2017).

\section{CONSIDERAÇÕES FINAIS}


O Engajamento Corporativo com Startups é uma modalidade de inovação aberta que tem ganhado enorme força num contexto de rápida evolução, difusão e acesso de tecnologias principalmente as digitais - em intercessão com o advento da nova revolução industrial. Números relativos a estes engajamentos são crescentes e apontam para um cenário em que ECcS estejam entre as principais formas de inovação aberta. Contudo, o campo ainda é embrionário e há muitas oportunidades de investigação e aprofundamentos na teoria e na prática.

O presente estudo é parte de um esforço inicial para se compreender como as grandes empresas se organizam - ou devem se organizar - para obterem melhor desempenho em seus engajamentos com startups. Um mapeamento da literatura orientado pelos princípios da revisão sistemática possibilitou identificar as principais vertentes que antecedem os estudos no tema e os principais dilemas. Foi evidenciado que os relatos e debates sobre ECcS são bastante recentes e carecem de maior interlocução com o campo da gestão da inovação com foco na organização interna da empresa. Tal interação pode enriquecer sobremaneira os debates prático e teórico, suportar a resolução de pontos de tensão e fornecer subsídios para arranjos de rede mais complexos e longevos em prol da inovação sistemática.

\section{AGRADECIMENTOS}

O presente trabalho foi realizado com apoio da Coordenação de Aperfeiçoamento de Pessoal de Nível Superior - Brasil (CAPES) - Código de Financiamento 001.

\section{REFERÊNCIAS}

ADAMS, R.; BESSANT, J.; PHELPS, R. Innovation management measurement: A review. International Journal of Management Reviews, v. 8, n. 1, p. 21-47, 2006.

ALÄNGE, S.; STEIBER, A. Three operational models for ambidexterity in large corporations. Triple Helix, v. 5 , n. 1, p. 5, 2018.

ARENA, M.; CROSS, R.; SIMS, J.; UHL-BIEN, M. How to catalyze innovation in your organization. MIT Sloan Management Review, v. 58, n. 4, p. 38-48, 2017.

BAGNO, R.B.; SALERNO, M.S.; AMATO NETO, J.; SILVA, D. Innovation and technological competence building in Brazilian automotive industry subsidiaries from the perspective of interorganizational networks. Proceedings of the 20th International GERPISA Colloquium, Krakow, 2012.

BAGNO, R.B.; SALERNO, M.S.; DIAS, A.V.C. Innovation as a new organizational function: evidence and characterization from large industrial companies in Brazil. Production, v. 27, 2017.

BAGNO, R.B.; SALERNO, M.S.; SILVA, D.O. Models with graphical representation for innovation management: a literature review. R\&D Management, v. 47, n. 4, p. 637-653, 2017.

BASU, S.; PHELPS, C.; KOTHA, S. Towards understanding who makes corporate venture capital investments and why. Journal of Business Venturing, v. 26, n. 2, p. 153-171, 2011. 
CHESBROUGH, H. Open Innovation: the New Imperative for Creating and Profiting from Technology. Boston: Harvard Business School Press, 2003.

CHIARONI, D.; CHIESA, V.; FRATTINI, F. The Open Innovation Journey: How firms dynamically implement the emerging innovation management paradigm. Technovation, v. 31, n. 1, p. 34-43, 2011.

DUNCAN, R.B. The ambidextrous organization: Designing dual structures for innovation. The management of organization, v. 1, p. 167-188, 1976.

FREEMAN, J.; ENGEL, J.S. Models of innovation: Startups and mature corporations. California Management Review, v. 50, n. 1, p. 94-119, 2007.

FREYTAG, R. Strategic negotiations: three essentials for successful partnerships with startups. Strategy \& Leadership, v. 47, n. 1, p. 19-25, 2019.

GREENWOOD, R.; MILLER, D. Tackling design anew: Getting back to the heart of organizational theory. Academy of Management Perspectives, v. 24, n. 4, p. 78-88, 2010.

HOGENHUIS, B.N.; VAN DEN HENDE, E.A.; HULTINK, E.J. When Should Large Firms Collaborate with Young Ventures? Understanding young firms' strengths can help firms make the right decisions around asymmetric collaborations. Research-Technology Management, v. 59, n. 1, p. 39-47, 2016.

HUIZINGH, E.K. Open innovation: State of the art and future perspectives. Technovation, v. 31, n. 1, p. 2-9, 2011.

HUMPHREY, J.; SCHMITZ, H. Governance and Upgrading in Global Value Chains, a background paper for the Bellagio Value Chain Workshop. IDS, University of Sussex, UK, 2000.

INNOVATION LEADER. Startup engagement: best practices for large organizations (report excerpt). Innovation Leader, p.21. 2019

KANBACH, D.K.; STUBNER, S. Corporate accelerators as recent form of startup engagement: The what, the why, and the how. Journal of Applied Business Research, v. 32, n. 6, p. 1761, 2016.

KAPLAN, S. How Big Companies \& Startups Use "Co-Creation" to Innovate. Inc. 2019 Disponível em: < https://www.inc.com/soren-kaplan/how-big-companies-amp-startups-use-co-creation-to-innovate.html >.Acesso em: 3 Mai 2019.

KELLEY, D. Adaptation and organizational connectedness in corporate radical innovation programs. Journal of product innovation management, v. 26, n. 5, p. 487-501, 2009.

KOHLER, T. Corporate accelerators: Building bridges between corporations and startups. Business Horizons, v. 59, n. 3, p. 347-357, 2016

LAWTON, J. What to expect from your corporate accelerator. Innovation Leader Pointers, n. June, p. 19-21, 2019.

LIVIERATOS, A.D.; LEPENIOTIS, P. Corporate venture capital programs of European electric utilities: Motives, trends, strategies and challenges. The Electricity Journal, v. 30, n. 2, p. 30-40, 2017.

MAHMOUD-JOUINI, S.B.; DUVERT, C.; ESQUIROL, M. Key Factors in Building a Corporate Accelerator Capability: Developing an effective corporate accelerator requires close attention to the relationships between startups and the sponsoring company. Research-Technology Management, v. 61, n. 4, p. 26-34, 2018.

MELO, J.C.F.; BAGNO, R.B. Atribuições de um time dedicado à gestão da inovação em um grupo industrial brasileiro: um estudo longitudinal baseado em eventos. Revista Produção Online, v. 17, n. 2, p. 482-506, 2017.

MINSHALL, T.; MORTARA, L.; VALLI, R.; PROBERT, D. Making "asymmetric" partnerships work. Research-Technology Management, v. 53, n. 3, p. 53-63, 2010.

MINSKY, C. Big corporates are only interested in "innovation theatre". Sifted 2019 Disponível em: < https://sifted.eu/articles/big-european-companies-startups-deloitte-survey/ >.Acesso em: 12 May 2019.

O'CONNOR, G.C. Innovation: from process to function. Journal of product innovation management, v. $29, \mathrm{n}$. 3, p. 361-363, 2012. 
O'CONNOR, G.C.; CORBETT, A.C.; PETERS, L.S. Beyond the champion: institutionalizing innovation through people. Stanford University Press, 2018.

O'CONNOR, G.C.; LEIFER, R.; PAULSON, A.S.; PETERS, L.S. Grabbing Lightning: Building a Capability for Breakthrough Innovation. San Francisco: John Wiley \& Sons, 2008. 332p.

O'REILLY, C.A.; TUSHMAN, M.L. The ambidextrous organization. Harvard business review, v. 82, n. 4, p. 74, 2004.

O'REILLY III, C.A.; TUSHMAN, M.L. Organizational ambidexterity: Past, present, and future. Academy of management Perspectives, v. 27, n. 4, p. 324-338, 2013.

PRASHANTHAM, S.; KUMAR, K. Engaging with startups: MNC Perspectives. IIMB Management Review, 2019.

PRASHANTHAM, S.; YIP, G.S. Engaging with startups in emerging markets. 2016.

RAISCH, S.; BIRKINSHAW, J. Organizational ambidexterity: Antecedents, outcomes, and moderators. Journal of management, v. 34, n. 3, p. 375-409, 2008.

RICHTER, N.; JACKSON, P.; SCHILDHAUER, T. Outsourcing creativity: An abductive study of open innovation using corporate accelerators. Creativity and Innovation Management, v. 27, n. 1, p. 69-78, 2018.

SELIG, C.J.; GASSER, T.; BALTES, G.H. How Corporate Accelerators Foster Organizational Transformation: An Internal Perspective. p. 1-9, 2018.

SHANKAR, R.K.; SHEPHERD, D.A. Accelerating strategic fit or venture emergence: Different paths adopted by corporate accelerators. Journal of Business Venturing, 2018.

SPENDER, J.-C.; CORVELLO, V.; GRIMALDI, M.; RIPPA, P. Startups and open innovation: a review of the literature. European Journal of Innovation Management, v. 20, n. 1, p. 4-30, 2017.

TIDD, J.; BESSANT, J.; PAVITT, K. Managing innovation integrating technological, market and organizational change. Chichester, UK: John Wiley and Sons, 2001.

WEIBLEN, T.; CHESBROUGH, H.W. Engaging with startups to enhance corporate innovation. California Management Review, v. 57, n. 2, p. 66-90, 2015. 